\title{
NANOSTRUCTURATION OF POLYAROMATIC ANALOGUES OF THE CARBONACEOUS DUST
}

\author{
Y. Carpentier ${ }^{1,2}$, G. Féraud ${ }^{1}$, E. Dartois ${ }^{3}$, R. Brunetto ${ }^{3}$, E. Charon ${ }^{4}$, \\ A.-T. Cao ${ }^{1,5}$, L. d'Hendecourt ${ }^{3}$, Ph. Bréchignac ${ }^{1}$, J.-N. Rouzaud ${ }^{4}$ \\ and T. Pino ${ }^{1}$
}

\begin{abstract}
Carbonaceous cosmic dust is mainly observed through infrared spectroscopy either in absorption or in emission. Laboratory soot analogues of this interstellar or circumstellar dust were produced in fuel-rich, low-pressure, premixed and flat, flames. The particles were investigated by infrared absorption spectroscopy in the $2-15 \mu \mathrm{m}$ spectral region. The details of the spectral features shed some light on the structure of the material and enable the study of its life cycle. In particular, the $8 \mu \mathrm{m}$ band position is tentatively attributed to defects at the edge or in the polyaromatic units of the materials, revealing these structural changes in astrophysical dust.
\end{abstract}

\section{Introduction}

Carbonaceous materials, from molecules to solid grains, are observed in many regions in space (Ehrenfreund \& Charnley 2000; Henning \& Salama 1998). Although carbonaceous materials carry spectral features observed in the visible to UV range (Désert et al. 1990; Draine 2003; Zubko et al. 2004), most of the spectral information is obtained in the infrared domain. In particular the mid-infrared emission features observed in many astronomical objects (Gillett et al. 1973), the Aromatic

\footnotetext{
${ }^{1}$ Institut des Sciences Moléculaires d'Orsay, CNRS - Univ. Paris Sud, UMR 8214, 91405 Orsay Cedex, France

2 Present address: Laboratoire de Physique des Laser, Atomes et Molécules, Bât. P5 USTL, 59655 Villeneuve d'Ascq Cedex, France

${ }^{3}$ Institut d'Astrophysique Spatiale, CNRS - Univ. Paris-Sud 11, UMR 8617, 91405 Orsay Cedex, France

${ }^{4}$ Laboratoire de Géologie, École Normale Supérieure, UMR CNRS 8538, 75231 Paris Cedex 5, France

${ }^{5}$ Present address: Physics Department, University of Pedagogy of Ho Chi Minh City, Ho Chi Minh City, Viet Nam
} 
Infrared Bands (AIBs), are tracing carbonaceous particles whose inferred size vary from large molecules, the Polycyclic Aromatic Hydrocarbons - PAHs, to nanoparticles of a few nanometers (Crawford et al. 1985; Léger \& Puget 1984; Tielens 2008). These carbonaceous species, heated by UV/visible starlight, emit through their vibrational bands thanks to the transient heating mechanism (Allamandola et al. 1989; Draine \& Li 2001; Puget \& Léger 1989). Three main classes A, B and $\mathrm{C}$ of astrophysical spectra have been proposed based on the observed spectral characteristics in the 6 to $9 \mu \mathrm{m}$ wavelength region (Peeters et al. 2002). The band positions, in most sources, are characteristic of aromatic materials (Goto et al. 2007), however, the exact nature of the emitters is still debated. The presence of polymeric hydrogenated amorphous carbon (a-C:H) is also given by the $3.4 \mu \mathrm{m}$ feature observed in absorption in the diffuse interstellar medium (ISM), and in emission in class $\mathrm{C}$ objects. Related bands at 6.85 and $7.25 \mu \mathrm{m}$ are observed as well (Dartois et al. 2007; Dartois \& Muñoz-Caro 2007; Dartois et al. 2004, 2005; Pendleton \& Allamandola 2002). A link between these two components, amorphous and more organised materials, is also expected and different scenarios have been proposed.

In order to study these materials, many studies in laboratory astrophysics are ongoing involving various analogues. At Orsay (France), our gas-phase chemical reactor is a low-pressure flame, flat and premixed. It is a model reactor for combustion studies as it provides a one-dimensional time-resolved burning medium. In this study, we show that our reactor is capable of producing a structural/chemical variety of materials under controlled conditions. We focus here on the use of Fourier Transform Infrared absorption spectra. The laboratory and astronomical IR spectral features are tentatively interpreted in the light of the soot structures.

\section{Laboratory astrophysics: On the spectroscopic parameters for soot}

The production of laboratory analogues of the interstellar and circumstellar matter opens the way to detailed spectroscopic and structural characterisations. It enables the connection between the nanostructure of the soot grain and the spectral features. Soot grains consist of agglomerated primary particles with diameters of the order of 5-30 nm. We list in Table 1 most of the soot that were produced using different gas phase reactors together with the analytical tools that were used.

The techniques that are used can provide some information on the nature of the chemical bonds (IR - infrared absorption, EELS - Electron Energy Loss Spectroscopy, Raman spectroscopy, C13 NMR - Nuclear Magnetic Resonance, GCMS - Gas Chromatography Mass Spectrometry), the structural organisation of the polyaromatic domain (HRTEM - High Resolution Transmission Electron Microscopy, XRD - X-ray Diffraction, Raman spectroscopy) and the graphitization index (HRTEM, XRD, Raman, EELS, UV-vis absorption) through different spectroscopic parameters. The graphitization index is related to the size distribution of the polyaromatic units and is a major parameter to characterize a polyaromatic material. The polyaromatic unit sizes are equal to about 1-2 nanometers maximum in soot (measurements by Alfè et al. 2009; Vander Wal et al. 2007) whereas 
Table 1. Principal laboratory studies using gas phase reactors to produce the polyaromatic analogues of the interstellar and circumstellar grains. The main diagnostics that were used are also indicated.

\begin{tabular}{|c|c|}
\hline Chemical Reactor & Diagnostics \\
\hline Laser pyrolysis & $\begin{array}{l}\text { IR spectroscopy IR and HRTEM (Galvez et al. } \\
\text { 2002; Herlin et al. 1998; Reynaud et al. 2001) }\end{array}$ \\
\hline Laser pyrolysis & $\begin{array}{l}\text { IR spectroscopy, mass spectometry, EELS, } \\
\text { HRTEM (Schnaiter et al. 1999) }\end{array}$ \\
\hline Laser pyrolysis & $\begin{array}{l}\text { HRTEM, IR, UV-Vis, GCMS (Jäger et al. } \\
2006 \text { ) }\end{array}$ \\
\hline $\begin{array}{l}\text { Condensation at high } \\
\text { temperature }\end{array}$ & $\begin{array}{l}\text { HRTEM, EELS, C13 NMR, IR-UV (Jäger } \\
\text { et al. 2009) }\end{array}$ \\
\hline $\begin{array}{l}\text { Condensation at high } \\
\text { temperature }\end{array}$ & IR, XRD (Biennier et al. 2009) \\
\hline Laser pyrolysis & $\begin{array}{l}\text { HRTEM, IR, UV-Vis, GCMS (Jäger et al. } \\
2006 \text { ) }\end{array}$ \\
\hline $\begin{array}{l}\text { Premix Flame at atmo- } \\
\text { spheric pressure }\end{array}$ & UV Absorption (Schnaiter et al. 1996) \\
\hline $\begin{array}{l}\text { Benzene flame at atmo- } \\
\text { spheric pressure }\end{array}$ & UV Absorption (Colangeli et al. 1995) \\
\hline $\begin{array}{l}\text { Premix and low pressure } \\
\text { flame }\end{array}$ & $\begin{array}{l}\text { IR Spectroscopy (Pino et al. 2008), Raman } \\
\text { Spectroscopy Brunetto et al. (2009) }\end{array}$ \\
\hline
\end{tabular}

most spectral parameters are built for units longer than $2-3 \mathrm{~nm}$, so that all these techniques can not be conclusive. Other concepts may be used, the main one concerns the organisation of these polyaromatic units: the organised domains range from two to, typically, four stacked polyaromatic layers (Alfè et al. 2009; Bockhorn 1994; Dippel et al. 1999; Homann 1998; Sadezky et al. 2005). These stacked polyaromatic units are forming the basic structural units, the BSUs. This concept of $\mathrm{BSU}$ is at the core of the analysis of all polyaromatic materials. The global arrangement of these subunits (orientation) and their proportion depend strongly on the soot itself and therefore on the burning conditions (Alfè et al. 2009).

\section{Spectral variations and grain nanostructures, astrophysical implications}

Infrared astronomical spectra are the sole information that can be used to decipher the intimate structure of the AIB carriers. Therefore only spectroscopic parameters extracted from the infrared bands can be used. We are thus exploring the details of the variations of the infrared bands within different soot samples using complementary Raman spectroscopy and HRTEM imagery (not shown here) in order to link the spectral features to specific structural properties of the polyaromatic 
units within the soot. The detailed analysis is reported elsewhere (Carpentier et al. 2012). In Figure 1, infrared absorption spectra of three soot samples (1 to 3) produced in different conditions are shown. We found that the evolution observed in this sample (from soot 1 to 3 ) is in fact rather similar to the evolution of spectral properties from the class $\mathrm{A}$ to $\mathrm{C}$ of the AIBs.

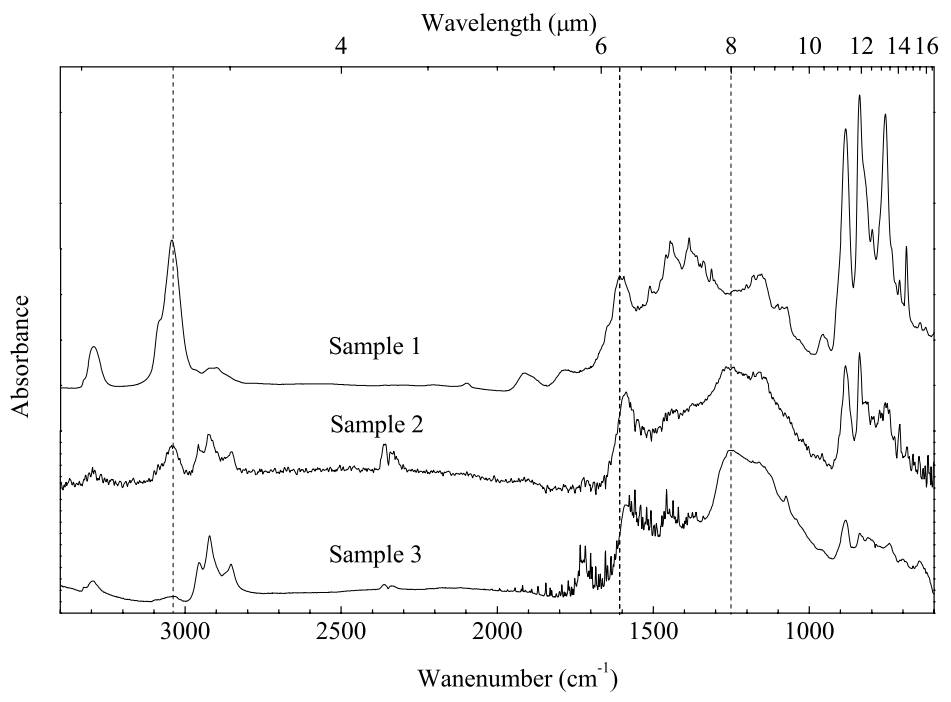

Fig. 1. IR absorption spectra of three soot samples after continuum subtraction. The spectra were normalized altogether in order to match the intensity of the band at $1600 \mathrm{~cm}^{-1}$. $\mathrm{CO}_{2}$ spectral contamination at about $2400 \mathrm{~cm}^{-1}$ for sample 2 and $\mathrm{H}_{2} \mathrm{O}$ spectral contamination in the $1800-1400 \mathrm{~cm}^{-1}$ range for sample 3 may be seen due to slight variations of the purge of the spectrometer. Note the shift of the band at about $1600 \mathrm{~cm}^{-1}$ and the presence or absence and shift of the band at about $1250 \mathrm{~cm}^{-1}$.

We have recently shown that the $6.2 \mu \mathrm{m}$ band position traces the intimate cross-linkage of the polyaromatic units composing the soot (Pino et al. 2008). Such evolution is accompanied by an evolution of the band at about $8 \mu \mathrm{m}$. We tentatively attribute this band to defect-like modes of the polyaromatic units, as outlined in Figure 1, either at the edge and cross-linking the units together or within the unit and shaping the unit away from planarity. Such nanostructural characteristics are, in combustion processes, related to the growth mechanism of the soot (D'Anna 2009). Similarly, different interstellar emitters could have experienced different formation pathways.

\section{Conclusion}

The study of soot analogues of the cosmic dust allows us to analyse the spectral characteristics of AIB spectra that were classified from A to C. The role of the 
defects in the carbonaceous particles appears to be at the core of the variations of the spectral features, in particular those related to the aromatic carbon skeleton, the band at about $6.2 \mu \mathrm{m}$ and that at about $8 \mu \mathrm{m}$. Progress on the understanding of the nanostructuration of the emitters will be essential to decipher the growth and lifecycle of this component of interstellar matter, as proposed in Carpentier et al. (2012).

This work has been supported by the French national program "Physique et Chimie du Milieu Interstellaire" and the French "Agence Nationale de la Recherche" (contract ANR-10-BLAN0502).

\section{References}

Alfè, M., Apicella, B., Barbella, R., et al., 2009, Proc. Comb. Inst., 32, 697

Allamandola, L.J., Tielens, A.G.G.M., \& Barker, J.R., 1989, ApJS, 71, 733

Biennier, L., Georges, R., Chandrasekaran, V., et al., 2009, Carbon, 47, 3295

Bockhorn, H. (ed.), 1994, Soot Formation in Combustion: Mechanisms and Models (Springer-Verlag, Heilderberg)

Brunetto, R., Pino, T., Dartois, E., et al., 2009, Icarus, 200, 323

Carpentier, Y., Féraud, G., Dartois, E., et al., 2012, A\&A, 548, A40

Colangeli, L., Mennella, V., Bussoletti, E., et al., 1995, Plan. Space. Sci., 43, 1263

Crawford, M., Tielens, A., \& Allamandola, L., 1985, ApJ, 293, L45

D'Anna, A., 2009, Combustion Generated Fine Carbonaceous particles, Chapt. Particle inception and growth: experimental evidences and a modelling attempt (KIT Scientific publishing)

Dartois, E., Geballe, T.R., Pino, T., et al., 2007, A\&A, 463, 635

Dartois, E., \& Muñoz-Caro, G.M., 2007, A\&A, 476, 1235

Dartois, E., Muñoz Caro, G.M., Deboffle, D., \& d'Hendecourt, L., 2004, A\&A, 423, L33

Dartois, E., Muñoz Caro, G.M., Deboffle, D., Montagnac, G., \& d'Hendecourt, L., 2005, A\&A, 432, 895

Désert, F.-X., Boulanger, F., \& Puget, J.L., 1990, A\&A, 237, 215

Dippel, B., Jander, H., \& Heintzenberg, J., 1999, Phys. Chem. Chem. Phys., 1, 4707

Draine, B.T., 2003, ARA\&A, 41, 241

Draine, B.T., \& Li, A., 2001, ApJ, 551, 807

Ehrenfreund, P., \& Charnley, S.B., 2000, ARA\&A, 38, 427

Galvez, A., Herlin-Boime, N., Reynaud, C., Clinard, C., \& Rouzaud, J.-N., 2002, Carbon, 40, 2775

Gillett, F.C., Forrest, W.J., \& Merrill, K.M., 1973, ApJ, 183, 87

Goto, M., Kwok, S., Takami, H., et al., 2007, ApJ, 662, 389

Henning, T., \& Salama, F., 1998, Science, 282, 2204

Herlin, N., Bohn, I., Reynaud, C., Cauchetier, M., Galvez, A., \& Rouzaud, J.-N., 1998, A\&A, 330, 1127

Homann, K.-H., 1998, Angew. Chem. Int. Ed. Engl., 37, 2434

Jäger, C., Huisken, F., Mutschke, H., Jansa, I.L., \& Henning, T., 2009, ApJ, 696, 706 
Jäger, C., Krasnokutski, S., Staicu, A., et al., 2006, ApJS, 166, 557

Léger, A., \& Puget, J., 1984, A\&A, 137, L5

Peeters, E., Hony, S., van Kerckhoven, C., et al., 2002, A\&A, 390, 1089

Pendleton, Y.J., \& Allamandola, L.J., 2002, ApJS, 138, 75

Pino, T., Dartois, E., Cao, A.-T., et al., 2008, A\&A, 490, 665

Puget, J.L., \& Léger, A., 1989, ARA\&A, 27, 161

Reynaud, C., Guillois, O., Herlin-Boime, N., et al., 2001, Spectrochim. Acta, 57, 797

Sadezky, A., Muckenhuber, H., Grothe, H., Niessner, R., \& Pöschl, U., 2005, Carbon, 43, 1731

Schnaiter, M., Henning, T., Mutschke, H., et al., 1999, ApJ, 519, 687

Schnaiter, M., Mutschke, H., Henning, T., et al., 1996, ApJ, 464, L187

Tielens, A.G.G.M., 2008, ARA\&A, 46, 289

Vander Wal, R.L., Yezerets, A., Currier, N.W., Kim, D.H., \& Wang, C.M., 2007, Carbon, 45,70

Zubko, V., Dwek, E., \& Arendt, R.G., 2004, ApJS, 152, 211 